\title{
Pacific
}

Journal of

Mathematics

\section{BIHOLOMORPHIC CONVEX MAPPINGS OF BALL IN $\mathbb{C}^{n}$}

Sheng Gong, ShI Kun Wang AND Qi HuANG Yu 


\title{
BIHOLOMORPHIC CONVEX MAPPINGS OF BALL IN $\mathbb{C}^{n}$
}

\section{Sheng Gong, Shikun Wang, AND Qihuang YU}

\begin{abstract}
Biholomorphic convex mappings from unit ball in $\mathbb{C}^{n}$ into $\mathbb{C}^{n}$ are studied in this paper. A Schwartz type lemma for the class of mappings and a necessary and sufficient condition under which a holomorphic mapping is biholomorphic and convex are established. The results are used to describe some characteristics of the image of the class of mappings.
\end{abstract}

1. Introduction. Since Loebe discovered as early as 1907 his "Verzerrugsatz", classical distortion theorems for families of univalent functions defined the unit disc in the complex plane $\mathbb{C}$ have developed systematically in depth and scope. For several variables, H. Cartan showed his interest in the field and conjectured [1] that the magnitude of the determinant of the complex Jacobian of a normalized biholomorphic mapping on the unit polydisc in $\mathbb{C}^{2}$ should have a finite upper and a positive lower bound. But, it was pointed out in [2] that the conjecture is not correct. That is the distortion theorem for general biholomorphic mappings in several variables does not hold. This suggests one has to find some of their subclasses for which the distortion theorem can still hold. In $1988 \mathrm{~S}$. Gong, C. H. FitzGerald and R. W. Bernard [3] obtained first time the upper and lower bound of the magnitude of the determinant of the Jacobian of a normalized, convex and biholomorphic mapping from the unit ball $B=\left\{Z=\left(z^{1}, z^{2}\right) \in \mathbb{C}^{2}|| Z \mid<1\right\}$ into $\mathbb{C}^{2}$. After that Taishun Liu [4] generalized the result to the unit ball in $\mathbb{C}^{n}$ and $X$. A. Zheng [5] to the bounded symmetric domains.

It is worth pointing out that the estimate of the magnitude of the determinant of the Jacobian of a holomorphic mapping is one of the generalization of distortion theorems of one variable. The estimation of eigenvalues of the Hermitian matrix, the product of the complex Jacobian of a holomorphic mapping and its transpose conjugate, should also be considered to be another form of generalization of distortion theorems of one complex variable. In this contribution we will discuss the type of distortion theorems and its application to biholomorphic convex mappings. 
The paper is arranged as follows: In $\S 2$ we will prove a Schwartz type lemma of biholomorphic convex mappings from the unit ball in $\mathbb{C}^{n}$ into $\mathbb{C}^{n}$. The lemma describes the type of distortion theorems given by us. In $\S 3$ we will give a necessary and sufficient condition under which a holomorphic mapping is biholomorphic and convex. In $[6,7]$ two necessary and sufficient conditions which are equivalent were also given. However, in the section we would quote a counterexample by Taishun Liu in [4] to illustrate that the conditions are not sufficient. In $\S 4$, as an application of the above results, we will study the geometric characteristics of the image of biholomorphic convex mappings.

In this paper summation convention is used.

2. A Schwartz type lemma of biholomorphic convex mappings. $B$ denotes the unit ball in $\mathbb{C}^{n}$, that is

$$
B=\left\{Z=\left.\left(z^{1}, \ldots, z^{n}\right) \in \mathbb{C}^{n}|| Z\right|^{2}=\sum_{i=1}^{n}\left|z^{i}\right|^{2}<1\right\} .
$$

When $n=1$ replace $B$ by the symbol $D$. The Jacobian of a mapping $f$ from $B$ to $\mathbb{C}^{n}$ is denoted by $J_{f}$. So-called normalized holomorphic mapping means the holomorphic one with $f(0)=0$ and $J_{f}(0)=I$, $I$ is the unit matrix.

In this section we demonstrate a Schwartz type lemma of biholomorphic convex mappings from $B$ into $\mathbb{C}^{n}$.

TheOREM 1. Let $f: B \rightarrow \mathbb{C}^{n}$ be a biholomorphic convex mapping. Then

$$
\left(\frac{1+|Z|}{1-|Z|}\right)^{2} g_{\alpha \bar{\beta}} d z^{\alpha} d \bar{z}^{\beta} \geq f^{*} \delta_{i j} d w^{i} d \bar{w}^{j} \geq\left(\frac{1-|Z|}{1+|Z|}\right)^{2} g_{\alpha \bar{\beta}} d z^{\alpha} d \bar{z}^{\beta}
$$

holds, where

$$
g_{\alpha \bar{\beta}}=\frac{\left(1-|Z|^{2}\right) \delta_{\alpha \beta}+\bar{z}^{\alpha} z^{\beta}}{\left(1-|Z|^{2}\right)^{2}}
$$

is the Bergman metric of the unit ball $B$ in $\mathbb{C}^{n}$ and the inequalities are sharp.

The proof of the theorem depends on the following two lemmas.

Lemma 1. Suppose that $F: B \rightarrow \mathbb{C}^{n}, W=F(\xi)$ is a biholomorphic convex mapping and $\xi=F^{-1}(W)$ is the inverse mapping of $F(\xi)$. 
Then

$$
\begin{aligned}
& \left.4 \delta_{\alpha \beta}|a| \frac{\partial \xi^{\alpha}}{\partial w^{i}} \frac{\partial \bar{\xi}^{\beta}}{\partial \bar{w}^{j}}\right|_{\xi=0} v^{i} \bar{v}^{j} \\
& \geq 2\left|\operatorname{Re}\left(\left.\delta_{\alpha \beta} \frac{\partial^{2} \xi^{\alpha}}{\partial w^{k} \partial w^{i}} \frac{\partial \bar{\xi}^{\beta}}{\partial \bar{w}^{j}} \frac{\partial w^{k}}{\partial z^{l}}\right|_{\xi=0} a^{l} v^{i} \bar{v}^{j}\right)\right|
\end{aligned}
$$

holds for any $a=\left(a^{1}, \ldots, a^{n}\right) \in B$ and $V=\left(v^{1}, \ldots, v^{n}\right) \in \mathbb{C}^{n}$, where $w^{i}(i=1,2, \ldots, n)$ are components of $W$ and $\xi^{\alpha}(\alpha=$ $1,2, \ldots, n)$ ones of $\xi$.

LEMMA 2. Suppose that $f: B \rightarrow \mathbb{C}^{n}, W=f(Z)$, is a biholomorphic convex mapping with $f(0)=0$ and $Z=f^{-1}(W)$ is the inverse mapping of $f(Z)$. Then

$$
\begin{aligned}
& 4|Z| g_{\alpha \bar{\beta}} \frac{\partial z^{\alpha}}{\partial w^{i}} \frac{\partial \bar{z}^{\beta}}{\partial \bar{w}^{j}} v^{i} \bar{v}^{j} \\
& \geq \mid 2 \operatorname{Re}\left\{g_{\beta \bar{\gamma}} \frac{\partial z^{\alpha}}{\partial w^{i}} \frac{\partial \bar{z}^{\gamma}}{\partial \bar{w}^{j}} \bar{z}^{\alpha} z^{\beta} v^{i} \bar{v}^{j}+|Z|^{2} g_{\alpha \bar{\beta}} \frac{\partial z^{\alpha}}{\partial w^{i}} \frac{\partial \bar{z}^{\beta}}{\partial \bar{w}^{j}} v^{i} \bar{v}^{j}\right. \\
&\left.+\left(1-|Z|^{2}\right) g_{\alpha \bar{\beta}} \frac{\partial^{2} z^{\alpha}}{\partial w^{k} \partial w^{i}} \frac{\partial \bar{z}^{\beta}}{\partial \bar{w}^{j}} \frac{\partial w^{k}}{\partial z^{\gamma}} z^{\gamma} v^{i} \bar{v}^{j}\right\} \mid
\end{aligned}
$$

holds for any $Z \in B, V=\left(v^{1}, \ldots, v^{n}\right) \in \mathbb{C}^{n}$.

Proof of Lemma 1. Since $F(B)$ is a convex set we have

$$
\lambda F(\xi)+(1-\lambda) F\left(\xi_{0}\right) \in F(B)
$$

for any $\lambda \in[0,1]$ and $\xi, \xi_{0} \in B$. Fix $\xi_{0}$ and define a holomorphic mapping $\xi_{\lambda}$ from $B$ to $B$ by

$$
\xi_{\lambda}=F^{-1}\left(\lambda F(\xi)+(1-\lambda) F\left(\xi_{0}\right)\right), \quad \xi \in B .
$$

From Schwartz's lemma of the unit ball in $\mathbb{C}^{n}$, it follows that

$$
g_{\alpha \bar{\beta}}(\xi) v^{\alpha} \bar{v}^{\beta} \geq g_{\alpha \bar{\beta}}\left(\xi_{\lambda}\right) \frac{\partial \xi_{\lambda}^{\alpha}}{\partial \xi^{k}} \frac{\partial \bar{\xi}_{\lambda}^{\beta}}{\partial \bar{\xi} l} v^{k} \bar{v}^{l}
$$

or

(1) $g_{\alpha \bar{\beta}^{\prime}}(\xi) v^{\alpha} \bar{v}^{\beta} \geq \lambda^{2} g_{\alpha \bar{\beta}^{\beta}}\left(\xi_{\lambda}\right) \frac{\partial \xi_{\lambda}^{\alpha}}{\partial w^{i}}\left(\xi_{\lambda}\right) \frac{\partial w^{i}}{\partial \xi^{k}}(\xi) \frac{\partial \bar{\xi}_{\lambda}^{\beta}}{\partial \bar{w}^{j}}\left(\xi_{\lambda}\right) \frac{\partial \bar{w}^{j}}{\partial \bar{\xi}^{l}}(\xi) v^{k} \bar{v}^{l}$ 
The inequality (1) can be rewritten as

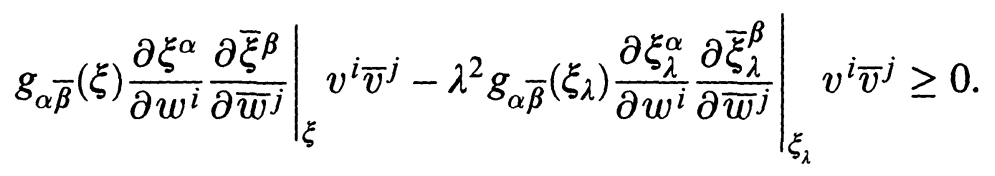

For $\lambda \in[0,1)$ we have

$$
\lim _{\lambda \rightarrow 1}\left[\frac{\left.g_{\alpha \bar{\beta}}(\xi) \frac{\partial \xi^{\alpha}}{\partial w^{i}} \frac{\partial \bar{\xi}^{\beta}}{\partial \bar{w}^{j}}\right|_{\xi} v^{i} \bar{v}^{j}-\left.\lambda^{2} g_{\partial \bar{\beta}}\left(\xi_{\lambda}\right) \frac{\partial \xi_{\lambda}^{\alpha}}{\partial w^{i}} \frac{\partial \bar{\xi}_{\lambda}^{\beta}}{\partial \bar{w}^{j}}\right|_{\xi_{\lambda}} v^{i} \bar{v}^{j}}{1-\lambda}\right] \geq 0
$$

Straightforward calculation yields

$$
\begin{aligned}
0 \leq \lim _{\lambda \rightarrow 1} & \frac{d}{d \lambda}\left[\left.\lambda^{2} g_{\alpha \bar{\beta}}\left(\xi_{\lambda}\right) \frac{\partial \xi_{\lambda}^{\alpha}}{\partial w^{i}} \frac{\partial \bar{\xi}_{\lambda}^{\beta}}{\partial \bar{w}^{j}}\right|_{\xi_{\lambda}} v^{i} \bar{v}^{j}\right] \\
=\lim _{\lambda \rightarrow 1} 2 & \left\{\left.\lambda g_{\alpha \bar{\beta}} \frac{\partial \xi_{\lambda}^{\alpha}}{\partial w^{i}} \frac{\partial \bar{\xi}_{\lambda}^{\beta}}{\partial \bar{w} j}\right|_{\xi_{\lambda}} v^{i} \bar{v}^{j}\right. \\
& +\operatorname{Re}\left[\left.\lambda^{2} g_{\alpha \bar{\beta}} \frac{\partial^{2} \xi_{\lambda}^{\alpha}}{\partial w^{k} \partial w^{i}} \frac{\partial \bar{\xi}_{\lambda}^{\beta}}{\partial \bar{w}^{j}}\right|_{\xi_{\lambda}}\left(w^{k}(\xi)-w^{k}\left(\xi_{0}\right)\right) v^{i} \bar{v}^{j}\right. \\
& \left.\left.+\left.\left.\lambda^{2} \frac{\partial \xi_{\lambda}^{\alpha}}{\partial w^{i}} \frac{\partial \bar{\xi}_{\lambda}^{\beta}}{\partial \bar{w}^{j}}\right|_{\xi_{\lambda}} v^{i} \bar{v}^{j} \frac{\partial g_{\alpha \bar{\beta}}}{\partial \xi_{\lambda}^{\gamma}} \frac{\partial \xi^{\gamma}}{\partial w^{k}}\right|_{\xi_{\lambda}}\left(w^{k}(\xi)-w^{k}\left(\xi_{0}\right)\right)\right]\right\} .
\end{aligned}
$$

Noting that $\xi_{\lambda} \rightarrow \xi$ as $\lambda \rightarrow 1$, we obtain at the point $\xi$

(2) $2 g_{\alpha \bar{\beta}} \frac{\partial \xi_{\lambda}^{\alpha}}{\partial w^{i}} \frac{\partial \bar{\xi}^{\beta}}{\partial \bar{w}^{j}} v^{i} \bar{v}^{j}$

$$
\begin{aligned}
+2 \operatorname{Re}\left\{g_{\alpha \bar{\beta}}\right. & \frac{\partial^{2} \xi^{\alpha}}{\partial w^{k} \partial w^{i}} \frac{\partial \bar{\xi}^{\beta}}{\partial \bar{w}^{j}}\left(w^{k}(\xi)-w^{k}\left(\xi_{0}\right)\right) v^{i} \bar{v}^{j} \\
& \left.+\frac{\partial \xi^{\alpha}}{\partial w^{i}} \frac{\partial \bar{\xi}^{\beta}}{\partial \bar{w}^{j}} v^{i} \bar{v}^{j} \frac{\partial g_{\alpha \bar{\beta}}}{\partial \xi^{\gamma}} \frac{\partial \xi^{\gamma}}{\partial w^{k}}\left(w^{k}(\xi)-w^{k}\left(\xi_{0}\right)\right)\right\} \geq 0 .
\end{aligned}
$$

The inequality (2) holds for any $\xi, \xi_{0} \in B$. Now fix $\xi$ and take $\xi_{0}=\frac{t \xi}{|\xi|}$ in (2) where $t \in D, D$ is the unit disc in $\mathbb{C}$. We then obtain 
a nonnegative harmonic function defined on the unit disc by

$$
\begin{aligned}
H(t)=2 g_{\alpha \bar{\beta}} \frac{\partial \xi^{\alpha}}{\partial w^{i}} \frac{\partial \bar{\xi}_{\lambda}^{\beta}}{\partial \bar{w}^{j}} v^{i} \bar{v}^{j} \\
+2 \operatorname{Re}\left\{g_{\alpha \bar{\beta}} \frac{\partial^{2} \xi^{\alpha}}{\partial w^{k} \partial w^{i}} \frac{\partial \bar{\xi}^{\beta}}{\partial \bar{w}^{j}}\left(w^{k}(\xi)-w^{k}\left(\frac{t \xi}{|\xi|}\right)\right) v^{i} \bar{v}^{j}\right. \\
\left.+\frac{\partial \xi^{\alpha}}{\partial w^{i}} \frac{\partial \bar{\xi}^{\beta}}{\partial \bar{w}^{j}} v^{i} \bar{v}^{j} \frac{\partial g_{\alpha \bar{\beta}}}{\partial \xi^{\gamma}} \frac{\partial \xi^{\gamma}}{\partial w^{k}}\left(2^{k}(\xi)-w^{k}\left(\frac{t \xi}{|\xi|}\right)\right)\right\} .
\end{aligned}
$$

It follows from Harnack's inequality that

$$
\frac{1-|t|}{1+|t|} H(0) \leq H(t) \leq H(0) \frac{1+|t|}{1-|t|} .
$$

Now taking $t=|\xi|$, we obtain

$$
\frac{1-|\xi|}{1+|\xi|} H(0) \leq H(|\xi|) \leq H(0) \frac{1+|\xi|}{1-|\xi|}
$$

or

$$
\frac{1-|\xi|}{1+|\xi|} H(|\xi|) \leq H(0) \leq H(|\xi|) \frac{1+|\xi|}{1-|\xi|}
$$

where

and

$$
H(|\xi|)=2 g_{\alpha \bar{\beta}} \frac{\partial \xi^{\alpha}}{\partial w^{i}} \frac{\partial \bar{\xi}^{\beta}}{\partial \bar{w}^{j}} v^{i} \bar{v}^{j}
$$

$$
\begin{aligned}
H(0)=2 g_{\alpha \bar{\beta}} \frac{\partial \xi^{\alpha}}{\partial w^{i}} \frac{\partial \bar{\xi}^{\beta}}{\partial \bar{w}^{j}} v^{i} \bar{v}^{j} \\
+2 \operatorname{Re}\left\{g_{\alpha \bar{\beta}} \frac{\partial^{2} \xi^{\alpha}}{\partial w^{k} \partial w^{i}} \frac{\partial \bar{\xi}^{\beta}}{\partial \bar{w}^{j}}(\xi)\left(w^{k}(\xi)-w^{k}(0)\right) v^{i} \bar{v}^{j}\right. \\
\left.+\frac{\partial \xi^{\alpha}}{\partial w^{i}} \frac{\partial \bar{\xi}^{\beta}}{\partial \bar{w}^{j}} v^{i} \bar{v}^{j} \frac{\partial g_{\alpha \bar{\beta}}}{\partial \xi^{\gamma}} \frac{\partial \xi^{\gamma}}{\partial w^{k}}(\xi)\left(w^{k}(\xi)-w(0)\right)\right\}
\end{aligned}
$$

The inequalities (3) can be rewritten as

$$
\begin{aligned}
& \frac{4|\xi|}{1-|\xi|} g_{\alpha \bar{\beta}} \frac{\partial \xi^{\alpha}}{\partial w^{i}} \frac{\partial \bar{\xi}^{\beta}}{\partial \bar{w}^{j}} v^{i} \bar{v}^{j} \\
& \geq 2 \operatorname{Re}\left\{g_{\alpha \bar{\beta}} \frac{\partial^{2} \xi^{\alpha}}{\partial w^{k} \partial w^{i}} \frac{\partial \bar{\xi}^{\beta}}{\partial \bar{w}^{j}} v^{i} \bar{v}^{j}\left(w^{k}(\xi)-w^{k}(0)\right)\right. \\
& \left.\quad+\frac{\partial \xi^{\alpha}}{\partial w^{i}} \frac{\partial \bar{\xi}^{\beta}}{\partial \bar{w}^{j}} v^{i} \bar{v}^{j} \frac{\partial g_{\alpha \bar{\beta}}}{\partial \xi^{\gamma}} \frac{\partial \xi^{\gamma}}{\partial w^{k}}\left(w^{k}(\xi)-w^{k}(0)\right)\right\} \\
& \geq \frac{-4|\xi|}{1+|\xi|} g_{\alpha \bar{\beta}} \frac{\partial \xi^{\alpha}}{\partial w^{i}} \frac{\partial \bar{\xi}^{\beta}}{\partial \bar{w}^{j}} v^{i} \bar{v}^{j} .
\end{aligned}
$$


Taking $\xi=\nu a, \nu \in(0,1)$, and letting $\nu \rightarrow 0$ in the inequalities above, we have

$$
\begin{aligned}
& \left.4|a| \delta_{\alpha \bar{\beta}} \frac{\partial \xi^{\alpha}}{\partial w^{i}} \frac{\partial \bar{\xi}^{\beta}}{\partial \bar{w}^{j}} v^{i} \bar{v}^{j}\right|_{\xi=0} \\
& \geq\left.\left|2 \operatorname{Re}\left(\delta_{\alpha \bar{\beta}} \frac{\partial^{2} \xi^{\alpha}}{\partial w^{k} \partial w^{i}} \frac{\partial \bar{\xi}^{\beta}}{\partial \bar{w}^{j}} \frac{\partial w^{k}}{\partial \xi^{l}} a^{l} v^{i} \bar{v}^{j}\right)\right|\right|_{\xi=0} .
\end{aligned}
$$

Lemma 1 is completely proved.

Proof of Lemma 2. Let $\varphi_{a}: B \rightarrow B$ be a holomorphic automorphism defined by

$$
\varphi_{a}(\xi)=\frac{a-\xi}{1-\xi \bar{a}^{\prime}} A,
$$

where $A=s I+\frac{\bar{a}^{\prime} a}{1+s}, s=\sqrt{1-|a|^{2}}, a \in B$. Denote $Z=\varphi_{a}(\xi)$. The inverse mapping of $\varphi_{a}$ is as follows:

$$
\xi=\varphi^{-1}(Z)=\frac{a-Z}{1-Z \bar{a}^{\prime}} A .
$$

Let $F(\xi)$ in Lemma 1 be $f\left(\varphi_{a}(\xi)\right)$. Straightforward calculation gives

$$
\begin{aligned}
&\left.\frac{\partial^{2} \xi^{\alpha}}{\partial w^{k} \partial w^{i}}\right|_{\xi=0}=\frac{\partial^{2} \xi^{\alpha}}{\partial z^{\mu} \partial z^{\nu}} \frac{\partial z^{\mu}}{\partial w^{k}} \frac{\partial z^{\nu}}{\partial w^{i}}+\frac{\partial \xi^{\alpha}}{\partial z^{\nu}} \frac{\partial^{2} z^{\nu}}{\partial w^{k} \partial w^{i}} \\
&=\frac{\bar{a}^{\mu} A_{\nu}^{\alpha}+\bar{a}^{\nu} A_{\mu}^{a}}{\left(1-|a|^{2}\right)^{2}} \frac{\partial z^{\nu}}{\partial w^{k}} \frac{\partial z^{\mu}}{\partial w^{i}}-\frac{A_{\mu}^{\alpha}}{1-|a|^{2}} \frac{\partial^{2} z^{\mu}}{\partial w^{k} \partial w^{i}}, \\
&\left.\frac{\partial \bar{\xi}^{\beta}}{\partial \bar{w}^{j}}\right|_{\xi=0}=-\frac{\bar{A}_{\sigma}^{\beta}}{1-|a|^{2}} \frac{\partial \bar{z}^{\sigma}}{\partial \bar{w}^{j}}, \\
&\left.\frac{\partial w^{k}}{\partial \xi^{l}}\right|_{\xi=0}=\left(\bar{a}^{l} a^{\tau}-A_{l}^{\tau}\right) \frac{\partial w^{k}}{\partial z^{\tau}} .
\end{aligned}
$$

Substituting (5) into the right side in (4) we have at $\xi=0(Z=a)$

$$
\begin{aligned}
2 \operatorname{Re}\left(\left.\delta_{\alpha \bar{\beta}} \frac{\partial^{2} \xi^{\alpha}}{\partial w^{k} \partial w^{i}} \frac{\partial \bar{\xi}^{\beta}}{\partial \bar{w}^{j}} \frac{\partial w^{k}}{\partial \xi^{l}}\right|_{\xi=0} a^{l} v^{i} \bar{v}^{j}\right) \\
=-2 \operatorname{Re}\left\{\left(\bar{a}^{\mu} g_{\nu \bar{\sigma}}+\bar{a}^{\nu} g_{\mu \bar{\sigma}}\right) a^{\nu} \frac{\partial z^{\mu}}{\partial w^{i}} \frac{\partial \bar{z}^{\sigma}}{\partial \bar{w}^{j}} v^{i} \bar{v}^{j}\right. \\
\left.+\left(1-|a|^{2}\right) g_{\mu \bar{\sigma}} \frac{\partial^{2} z^{\mu}}{\partial w^{k} \partial w^{j}} \frac{\partial \bar{z}^{\sigma}}{\partial \bar{w}^{\gamma}} \frac{\partial w^{k}}{\partial z^{\tau}} v^{i} \bar{v}^{j}\right\}
\end{aligned}
$$


Notice that the metric $\left(g_{\alpha \bar{\beta}}\right)_{1 \leq \alpha, \beta \leq n}$ on $B$ is invariant under its holomorphic automorphism. That is to say

$$
\left.\delta_{\alpha \beta} \frac{\partial \xi^{\alpha}}{\partial w^{i}} \frac{\partial \bar{\xi}^{\beta}}{\partial \bar{w}^{j}}\right|_{\xi=0} v^{i} \bar{v}^{j}=\left.g_{\alpha \bar{\beta}}(a) \frac{\partial z^{\alpha}}{\partial w^{i}} \frac{\partial \bar{z}^{\beta}}{\partial \bar{w}^{j}}\right|_{Z=a} v^{i} \bar{v}^{j}
$$

Combining (6), (7) with (4), we obtain at $Z=a$.

(8) $4|a| g_{\alpha \bar{\beta}} \frac{\partial z^{\alpha}}{\partial w^{i}} \frac{\partial \bar{z}^{\beta}}{\partial \bar{w}^{j}} v^{i} \bar{v}^{j}$

$$
\begin{aligned}
\geq \mid 2 \operatorname{Re}\{ & \bar{a}^{\alpha} a^{\beta} g_{\beta \bar{\gamma}} \frac{\partial z^{\alpha}}{\partial w^{i}} \frac{\partial \bar{z}^{\gamma}}{\partial \bar{w}^{j}} \bar{z}^{\alpha} z^{\beta} v^{i} \bar{v}^{j} \\
& +|a|^{2} g_{\alpha \bar{\beta}} \frac{\partial z^{\alpha}}{\partial w^{i}} \frac{\partial \bar{z}^{\beta}}{\partial \bar{w}^{j}} v^{i} \bar{v}^{j} \\
& \left.+\left(1-|a|^{2}\right) g_{\alpha \bar{\beta}} \frac{\partial^{2} z^{\alpha}}{\partial w^{k} \partial w^{i}} \frac{\partial \bar{z}^{\beta}}{\partial \bar{w}^{j}} \frac{\partial w^{k}}{\partial z^{\gamma}} a^{\gamma} v^{i} \bar{v}^{j}\right\} \mid
\end{aligned}
$$

The inequality (8) is valid for any $a \in B$. we complete the proof of Lemma 2.

Proof of Theorem 1. From the inequality (8) it follows that

(9) $\quad 2|Z|(2-|Z|) g_{\alpha \bar{\beta}} \frac{\partial \bar{z}^{\alpha}}{\partial w^{i}} \frac{\partial \bar{z}^{\beta}}{\partial \bar{w}^{j}} v^{i} \bar{v}^{j}$

$$
\begin{aligned}
\geq 2 \operatorname{Re} & \left\{\bar{z}^{\alpha} z^{\beta} g_{\beta \bar{\gamma}} \frac{\partial z^{\alpha}}{\partial w^{i}} \frac{\partial \bar{z}^{\gamma}}{\partial \bar{w}^{j}} v^{i} \bar{v}^{j}\right. \\
& \left.+\left(1-|Z|^{2}\right) g_{\alpha \bar{\beta}} \frac{\partial^{2} z^{\alpha}}{\partial w^{k} \partial w^{i}} \frac{\partial \bar{z}^{\beta}}{\partial \bar{w}^{j}} \frac{\partial w^{k}}{\partial z^{\gamma}} z^{\gamma} v^{i} \bar{v}^{j}\right\},
\end{aligned}
$$

for any $V \in \mathbb{C}^{n}$. Using the definition of $g_{\beta \bar{\gamma}}$ we have

$$
z^{\beta} g_{\beta \bar{\gamma}}=\frac{z^{\gamma}}{\left(1-|Z|^{2}\right)^{2}}
$$

and it is easy to see that the second term of the right side in (9) 
(11)

$$
\begin{aligned}
\left(1-|Z|^{2}\right) g_{\alpha \bar{\beta}} \frac{\partial^{2} z^{\alpha}}{\partial w^{k} \partial w^{i}} \frac{\partial \bar{\xi}^{\beta}}{\partial \bar{w}^{j}} \frac{\partial w^{k}}{\partial z^{\gamma}} z^{\gamma} v^{i} \bar{v}^{j} \\
=z^{\gamma} \frac{\partial}{\partial z^{\gamma}}\left\{\left(1-|Z|^{2}\right) g_{\alpha \bar{\beta}} \frac{\partial z^{\alpha}}{\partial w^{i}} \frac{\partial \bar{z}_{\beta}}{\partial \bar{w}^{j}} v^{i} \bar{v}^{j}\right\} \\
+|Z|^{2} g_{\alpha \bar{\beta}} \frac{\partial z^{\alpha}}{\partial w^{i}} \frac{\partial \bar{z}^{\beta}}{\partial \bar{w}^{j}} v^{i} \bar{v}^{j} \\
-\frac{\partial z^{\alpha}}{\partial w^{i}} \frac{\partial \bar{z}^{\beta}}{\partial \bar{w}^{j}} v^{i} \bar{v}^{j}\left[|Z|^{2} g_{\alpha \bar{\beta}}+\frac{\bar{z}^{\beta} z^{\alpha}}{\left(1-|Z|^{2}\right)^{2}}\right] \\
=z^{\gamma} \frac{\partial}{\partial z^{\gamma}}\left[\left(1-|Z|^{2}\right) g_{\alpha \bar{\beta}} \frac{\partial z^{\alpha}}{\partial w^{i}} \frac{\partial \bar{z}^{\beta}}{\partial \bar{w}^{j}} v^{i} \bar{v}^{j}\right] \\
-\frac{\bar{z}^{\alpha} z^{\beta}}{\left(1-|Z|^{2}\right)^{2}} \frac{\partial z^{\alpha}}{\partial w^{i}} \frac{\partial \bar{z}^{\beta}}{\partial \bar{w}^{j}} v^{i} \bar{v}^{j} .
\end{aligned}
$$

Substituting (10), (11) into (9) immediately gives

$$
\begin{aligned}
& 2|Z|(2-|Z|) g_{\alpha \bar{\beta}} \frac{\partial z^{\alpha}}{\partial w^{i}} \frac{\partial \bar{z}^{\beta}}{\partial \bar{w}^{j}} v^{i} \bar{v}^{j} \\
& \quad \geq 2 \operatorname{Re}\left\{z^{\gamma} \frac{\partial}{\partial z^{\gamma}}\left[\left(1-|Z|^{2}\right) g_{\alpha \bar{\beta}} \frac{\partial z^{\alpha}}{\partial w^{i}} \frac{\partial \bar{z}^{\beta}}{\partial \bar{w}^{j}} v^{i} \bar{v}^{j}\right]\right\} .
\end{aligned}
$$

Noting the Euclidean inner product $\left\langle d z^{\alpha}, d z^{\beta}\right\rangle=2 \delta_{\alpha \bar{\beta}},\left\langle d z^{\alpha}, d \bar{z}^{\beta}\right\rangle$ $=0$, we easily see that the right side in (12)

$$
\begin{aligned}
2 \operatorname{Re} & \left\{z^{\gamma} \frac{\partial}{\partial z^{\gamma}}\left[\left(1-|Z|^{2}\right) g_{\alpha \bar{\beta}} \frac{\partial z^{\alpha}}{\partial w^{i}} \frac{\partial \bar{z}^{\beta}}{\partial \bar{w}^{j}} v^{i} \bar{v}^{j}\right]\right\} \\
& =\frac{1}{2}\left\langle d\left[\left(1-|Z|^{2}\right) g_{\alpha \bar{\beta}} \frac{\partial z^{\alpha}}{\partial w^{i}} \frac{\partial \bar{z}^{\beta}}{\partial \bar{w}^{j}} v^{i} \bar{v}^{j}\right], d|Z|^{2}\right\rangle .
\end{aligned}
$$

By substituting (13) into (12), it follows that

$$
\begin{aligned}
2|Z| & (2-|Z|) g_{\alpha \bar{\beta}} \frac{\partial z^{\alpha}}{\partial w^{i}} \frac{\partial \bar{z}^{\beta}}{\partial \bar{w}^{j}} v^{i} \bar{v}^{j} \\
& \geq \frac{1}{2}\left\langle d\left[\left(1-|Z|^{2}\right) g_{\alpha \bar{\beta}} \frac{\partial z^{\alpha}}{\partial w^{i}} \frac{\partial \bar{z}^{\beta}}{\partial \bar{w}^{j}} v^{i} \bar{v}^{j}\right], d|Z|^{2}\right\rangle .
\end{aligned}
$$

The inequality (14) can be written as

(15) $\frac{2(2-|Z|)}{1-|Z|^{2}} \geq\left\langle d\left\{\lg \left[\left(1-|Z|^{2}\right) g_{\alpha \bar{\beta}} \frac{\partial z^{\alpha}}{\partial w^{i}} \frac{\partial \bar{z}^{\beta}}{\partial \bar{w}^{j}} v^{i} \bar{v}^{j}\right]\right\}, d|Z|\right\rangle$. 
Hence, integrating both sides of (15) one obtains

$$
\int_{0}^{|Z|} \frac{2(2-r)}{1-r^{2}} d r \geq \lg \left[\left(1-|Z|^{2}\right) g_{\alpha \bar{\beta}} \frac{\partial z^{\alpha}}{\partial w^{i}} \frac{\partial \bar{z}^{\beta}}{\partial \bar{w}^{j}} v^{i} \bar{v}^{j}\right]-\lg |V|^{2}
$$

So

$$
\frac{(1+|Z|)^{3}}{(1-|Z|)}|V|^{2} \geq\left(1-|Z|^{2}\right) g_{\alpha \bar{\beta}} \frac{\partial z^{\alpha}}{\partial w^{i}} \frac{\partial \bar{z}^{\beta}}{\partial \bar{w}^{j}} v^{i} \bar{v}^{j}
$$

(16) implies that for any $Z \in B$ the following matrix

$$
\frac{(1+|Z|)^{3}}{1-|Z|} I-\left(1-|Z|^{2}\right)\left(g_{\alpha \bar{\beta}} \frac{\partial z^{\alpha}}{\partial w^{i}} \frac{\partial \bar{z}^{\beta}}{\partial \bar{w}^{j}}\right)_{1 \leq i, j \leq n}
$$

is positive semi-definite. So we get the following matrix inequality

$$
\left(\frac{1-|Z|}{1+|Z|}\right)^{2}\left(g_{\alpha \bar{\beta}}\right)_{1 \leq \alpha, \beta \leq n} \leq\left(\delta_{i j} \frac{\partial w^{i}}{\partial z^{\alpha}} \frac{\partial \bar{w}^{j}}{\partial \bar{z}^{\beta}}\right)_{1 \leq \alpha, \beta \leq n} .
$$

Similarly, we can prove

$$
\left(\frac{1-|Z|}{1+|Z|}\right)^{2}\left(g_{\alpha \bar{\beta}}\right)_{1 \leq \alpha, \beta \leq n} \geq\left(\delta_{i j} \frac{\partial w^{i}}{\partial z^{\alpha}} \frac{\partial \bar{w}^{j}}{\partial \bar{z}^{\beta}}\right)_{1 \leq \alpha, \beta \leq n}
$$

Combining (17) with (18), we complete the proof of the inequalities in Theorem 1.

Now let us give an extreme example to illustrate that the inequalities in Theorem 1 are sharp. Set $n=2$ and let

$$
W=f(Z)=f^{1}\left(z^{1}, z^{2}\right), \quad f^{1}\left(z^{1}, z^{2}\right)=\left(\frac{z^{1}}{1-z^{1}}, \frac{z^{2}}{1-z^{1}}\right) .
$$

It is easy to check that the mapping is normalized, convex and biholomorphic and its Jacobian is

$$
J_{f}(Z)=\left(\begin{array}{cc}
\frac{1}{\left(1-z^{1}\right)^{2}} & 0 \\
\frac{z^{2}}{\left(1-z^{1}\right)^{2}} & \frac{1}{1-z^{1}}
\end{array}\right)
$$

Take $V=(1,0)$. Then

$$
V J_{f}(Z)=V\left(\frac{\partial W}{\partial Z}\right)=\left(\frac{1}{\left(1-z^{1}\right)^{2}}, 0\right)
$$

and

$$
\begin{aligned}
& V J_{f}(Z){\overline{J_{f}(Z)}}^{\prime} \bar{V}^{\prime} \\
& \quad=\frac{1}{\left(1-z^{1}-\bar{z}^{1}+\left|z^{1}\right|^{2}\right)^{2}}=\frac{1}{\left(1-2 r_{1} \cos \theta_{1}+r_{1}^{2}\right)^{2}}
\end{aligned}
$$


where $z^{1}=r_{1} e^{i \theta_{1}}$. On the other hand, we have

$$
\begin{aligned}
& \left(\frac{1-|Z|}{1+|Z|}\right)^{2} V\left(g_{\alpha \bar{\beta}}\right)_{\alpha, \beta=1,2} \bar{V}^{\prime}=\frac{1-\left|z^{2}\right|^{2}}{(1+|Z|)^{4}}, \\
& \left(\frac{1+|Z|}{1-|Z|}\right)^{2} V\left(g_{\alpha \bar{\beta}}\right)_{\alpha, \beta=1,2} \bar{V}^{\prime}=\frac{1-\left|z^{2}\right|^{2}}{(1-|Z|)^{4}} .
\end{aligned}
$$

If $z^{2}=0, z^{1}=r_{1}\left(\theta_{1}=0\right)$, we obtain from (19) and (20)

$$
V J_{f}(Z) \overline{J_{f(Z)}} \bar{V}^{\prime}=\left(\frac{1+|Z|}{1-|Z|}\right)^{2} V\left(g_{\alpha \bar{\beta}}\right)_{\alpha, \beta=1,2} \bar{V}^{\prime} \text {. }
$$

If $z^{2}=0, z^{1}=-r_{1}\left(\theta_{1}=\pi\right)$, then from (19) and (21) we get

$$
V J_{f}(Z) \overline{J_{f}(Z)^{\prime}} \bar{V}^{\prime}=\left(\frac{1-|Z|}{1+|Z|}\right)^{2} V\left(g_{\alpha \bar{\beta}}\right)_{\alpha, \beta=1,2} \bar{V}^{\prime} .
$$

The two inequalities (22) and (23) imply that the inequalities in Theorem 1 are sharp.

3. Necessary and sufficient conditions of biholomorphic convex mappings. Denote $B_{r}=\left\{Z \in \mathbb{C}^{n}|| Z \mid<r<1\right\}$ and the boundary of $B_{r}$ by $S_{r}$. We will demonstrate the following theorem.

THEOREM 2. If $f: B \rightarrow \mathbb{C}^{n}$ is a holomorphic mapping with $f(0)=0$ and $\left|\operatorname{det} J_{f}(Z)\right|>0$, then the following statements are equivalent.

(i) $f$ is a biholomorphic convex mapping.

(ii) If $Z \in B$ and $b=\left(b^{1}, \ldots, b^{n}\right) \in \mathbb{C}^{n}$ satisfy $\operatorname{Re}\left(b^{\alpha} \bar{z}^{\alpha}\right)=0$, then

$$
|b|^{2}+\operatorname{Re}\left(b^{\beta} b^{\gamma} \frac{\partial w^{i}}{\partial z^{\beta}} \frac{\partial \bar{w}^{j}}{\partial z^{\gamma}} z^{\alpha} \frac{\partial^{2} z^{\alpha}}{\partial w^{i} \partial w^{j}}\right) \geq 0
$$

Proof. First prove (ii) from (i).

Since $f(B)$ is a convex set, the mapping

$$
Z_{\lambda}=f^{-1}\left(\lambda f\left(Z_{1}\right)+(1-\lambda) f\left(Z_{2}\right)\right),
$$

$Z_{1}, Z_{2} \in B$ and $0 \leq \lambda \leq 1$, defines a holomorphic mapping from $B \times B \rightarrow B$. Denote Carathéodory distance of the two points $P$ and $Q$ of a domain $\Omega$ in $\mathbb{C}^{n}$ by $\mathbb{C}_{\Omega}(P, Q)$. Writing $F\left(Z_{1}, Z_{2}\right)=Z_{\lambda}$ we see [8]

$$
\begin{aligned}
C_{B}\left(F\left(Z_{1}, Z_{2}\right), 0\right) & \leq C_{B \times B}\left(\left(Z_{1}, Z_{2}\right),(0,0)\right) \\
& =\max \left\{C_{B}\left(Z_{1}, 0\right), C_{B}\left(Z_{2}, 0\right)\right\}
\end{aligned}
$$


by the contraction property of Carathéodory distance for holomorphic mappings. Recalling the Carathéodory distance on the unit ball in $\mathbb{C}^{n}$

$$
C_{B}(Z, 0)=\frac{1}{2} \lg \frac{1+|Z|}{1-|Z|}, \quad Z \in B,
$$

then one has

$$
\left|Z_{\lambda}\right| \leq \max \left\{\left|Z_{1}\right|,\left|Z_{2}\right|\right\} \text {. }
$$

It implies that for any $\lambda \in(0,1)$ and $Z_{1}, Z_{2} \in B_{r}, Z_{\lambda} \in B_{r}$. Thus $f\left(B_{r}\right)$ is also a convex set. Since, moreover, $f$ is biholomorphic the image $f\left(S_{r}\right)$ (or $\partial f\left(B_{r}\right)$ ) of $S_{r}$ under the mapping $f$ is a real convex hypersurface. The second fundamental form $S$ of the hypersurface is positive semi-definite. In other words, for any tangent vector $U$ to $f\left(S_{r}\right)$, we have

$$
S(U, U) \geq 0 .
$$

Note that $f\left(S_{r}\right)$ is an isothermal surface of the function $r^{2}=|Z|^{2}$ 。 $f^{-1}$ and

$$
\operatorname{grad} r^{2}=\frac{\partial r^{2}}{\partial w^{i}} \frac{\partial}{\partial \bar{w}^{i}}+\frac{\partial r^{2}}{\partial \bar{w}^{i}} \frac{\partial}{\partial w^{i}}=\bar{z}^{\alpha} \frac{\partial z^{\alpha}}{\partial w^{i}} \frac{\partial}{\partial \bar{w}^{i}}+z^{\alpha} \frac{\partial \bar{z}^{\alpha}}{\partial \bar{w}^{i}} \frac{\partial}{\partial w^{i}}
$$

is a normal vector to $f\left(S_{r}\right)$. Denote the normal vector by $\nu \cdot \frac{\nu}{|\nu|}$ is a unit normal vector. Assume that $U=a^{i} \frac{\partial}{\partial w^{i}}+\bar{a}^{i} \frac{\partial}{\partial \bar{w}^{i}}$ with

$$
\operatorname{Re}\left(a^{i} \bar{z}^{\alpha}\left(\partial z^{\alpha} / \partial w^{i}\right)\right)=0
$$

Then we have

$$
S(U, U)=\left\langle\widetilde{D}_{U} \frac{\nu}{|\nu|}, U\right\rangle
$$

where $\widetilde{D}$ is the connection in $R^{2 n}$ (directional derivative). Via $\langle\nu, U\rangle$ $=0$ we have

$$
\left\langle\widetilde{D}_{U} \frac{\nu}{|\nu|}, U\right\rangle=\frac{1}{|\nu|}\left\langle\widetilde{D}_{U} \nu, U\right\rangle
$$

Hence, the above inequality (25) can be rewritten as

$$
\frac{1}{|\nu|}\left(a^{i} \bar{a}^{j} \frac{\partial^{2} r^{2}}{\partial w^{i} \partial \bar{w}^{j}}+\operatorname{Re}\left(a^{i} a^{j} \frac{\partial^{2} r^{2}}{\partial w^{i} \partial w^{j}}\right)\right) \geq 0 \text {. }
$$

Now set $b=a \frac{\partial Z}{\partial w}$ or $a^{i}=b^{\beta}\left(\partial w^{i} / \partial z^{\beta}\right)$. From $\operatorname{Re}\left(a^{i} z^{\bar{z}^{\beta}}\left(\partial z^{\beta} / \partial w^{i}\right)\right)$ $=0$, it follows that $\operatorname{Re}\left(b^{\beta} \bar{z}^{\beta}\right)=0$. Substituting $a^{i}=b^{\beta}\left(\partial w^{i} / \partial z^{\beta}\right)$ into (26) we can get (24).

Now prove (i) from the condition (ii). We divide the proof into three steps. 
In the first step, we show that if the mapping $f$ is injective on $B_{\mu}(0<\mu<1)$, then $f\left(B_{\mu}\right)$ is a convex set. In fact, since $f$ is an injection on $B_{\mu}, f\left(S_{r}\right)$ is a real hypersurface in $\mathbb{C}^{n}$ for any $r<\mu$. If $U=a^{i}\left(\partial / \partial w^{i}\right)+\bar{a}^{i}\left(\partial / \partial \bar{w}^{i}\right)$ is a real tangent vector to $f\left(S_{r}\right)$, the second fundamental form is

$$
\begin{aligned}
S(U, U) & =\frac{1}{|\nu|}\left(a^{i} \bar{a}^{j} \frac{\partial^{2} r^{2}}{\partial w^{i} \partial \bar{w}^{j}}+\operatorname{Re}\left(a^{i} a^{j} \frac{\partial^{2} r^{2}}{\partial w^{i} \partial w^{j}}\right)\right) \\
& =\frac{1}{|\nu|}\left(a^{i} \bar{a}^{j} \frac{\partial z^{\alpha}}{\partial w^{i}} \frac{\partial \bar{z}^{\alpha}}{\partial \bar{w}^{j}}+\operatorname{Re}\left(a^{i} a^{j} z^{\alpha} \frac{\partial^{2} z^{\beta}}{\partial w^{i} \partial w^{j}}\right)\right) .
\end{aligned}
$$

Set $b=a \frac{\partial Z}{\partial W}$. We have $\operatorname{Re}\left(b^{\beta} \bar{z}^{\beta}\right)=0$. Recalling the condition (ii) we get

$$
S(U, U) \geq 0 \text {. }
$$

Hence, $f\left(S_{r}\right)$ is a real convex hypersurface for any $r<\mu$, and then we conclude that $f\left(B_{\mu}\right)$ is a convex set.

In the second step, we prove if the mapping $f$ is injective on $B_{\mu}$, then $f$ is also injective on $\bar{B}_{\mu}$. Since $f\left(B_{\mu}\right)$ is a convex set $f\left(B_{\mu}\right)$ is starlike with respect to the origin. Therefore, we have

$$
\left\langle\left. d r^{2}\right|_{B_{\mu}}, d \rho^{2}\right\rangle \geq 0
$$

thanks to the necessary condition for starlike mappings [9], where $\rho^{2}=|W|^{2}$. If the statement is not true then there are at least two distinct points $X, Y \in \bar{B}_{\mu}$ such that $f(X)=f(Y)$. We know, for all $0 \leq s \leq 1, s f(X), s f(Y) \in f\left(\bar{B}_{\mu}\right)$. Because $f$ is a holomorphic immersion one can obtain the curve $X(s)$ with $X(1)=X$ which falls in $B$ by the method of analytic continuation such that $f(X(s))=$ $s f(X)$. That is $X(s)=f^{-1}(s f(X))$ is a univalent component of the inverse images of the segment $s f(X)$. Since

$$
\frac{d r^{2}(X(s))}{d s}=\left.\frac{1}{s}\left\langle d r^{2}(X(s)), d \rho\right\rangle\right|_{W=s f(X)} \geq 0
$$

for $0 \leq s \leq 1$, we have

$$
r^{2}(X(s)) \leq r^{2}(X(1))=r^{2}(X)=\mu .
$$

Thus

$$
X(s) \in \bar{B}_{\mu} .
$$

Suppose that $Y(s)$ is another univalent component of the inverse images of the segment $s f(X)$, but $Y(1)=Y$. A similar discussion shows $Y(s) \in \bar{B}_{\mu}$. Let

$$
\mathscr{R}=\{s \in[0,1] \mid X(s)=Y(s)\} .
$$


If the set $\mathscr{R}$ is nonempty then the supremum $s^{*}$ of $\mathscr{R}$ exists. Because $\mathscr{R}$ is a closed set $s^{*} \in \mathscr{R}$ and $s^{*}<1$ due to $X(1) \neq Y(1)$. This implies that for any small positive $\varepsilon$ satisfying $s^{*}<s^{*}+\varepsilon \leq 1$ we have $X\left(s^{*}+\varepsilon\right) \neq Y\left(s^{*}+\varepsilon\right)$. But

$$
f\left(X\left(s^{*}+\varepsilon\right)\right)=f\left(Y\left(s^{*}+\varepsilon\right)\right)=\left(s^{*}+\varepsilon\right) f(X) .
$$

This is contrary to $f$ being locally biholomorphic at $X\left(s^{*}\right)$. If $\mathscr{R}$ is empty then $X(0) \neq Y(0)$. In other words, at this case we have at least two points $X(0)$ and $Y(0)$ such that $f(X(0))=f(Y(0))=0$. Since $f$ is an injection on $B_{\mu}$, at least one of the two points $X(0)$ and $Y(0)$ must be a boundary point of $\bar{B}_{\mu}$. Suppose that $X(0) \in \partial \bar{B}_{\mu}$. Let $B(X(0), \delta)$ be the open ball central at $X(0)$ with radius $\delta$, which is so small that $B(X(0), \delta) \cap \mathscr{U}_{0}$ is empty, where $\mathscr{U}_{0} \subset B_{\mu}$ is the neighborhood of the origin in $B$ such that $f$ is biholomorphic on it. Because of the open mapping theorem $f\left(B(X(0), \delta) \cap B_{\mu}\right)$ is an open set and $f\left(\mathscr{U}_{0}\right)$ an open set including the origin of $\mathbb{C}^{n}$. So the origin is also a boundary point of the open set $f\left(B(X(0), \delta) \cap B_{\mu}\right)$ and then $\left\{f\left(B(X(0), \delta) \cap B_{\mu}\right)\right\} \cap\left\{f\left(\mathscr{U}_{0}\right)\right\}$ is not empty. This implies that for any $W \in\left\{f\left(B(X(0), \delta) \cap B_{\mu}\right)\right\} \cap\left\{f\left(\mathscr{U}_{0}\right)\right\}$ it has two distinguished inverse images on $B_{\mu}$. It is impossible due to the fact that $f$ is injective on $B_{\mu}$. So, $f$ is also an injection on $\bar{B}_{\mu}$.

In the third step, we demonstrate $\mathscr{A}=[0,1]$ where $\mathscr{A}=\{t \in$ $[0,1] \mid f$ is injective on $\left.B_{t}\right\}$. Since $f(0)=0, J_{f}(0) \neq 0$, the set $\mathscr{A}$ is nonempty. We claim that $\mathscr{A}$ is a closed set. In fact, if $0<t_{1} \in \mathscr{A}$ then all $t \leq t_{1}$ fall in $\mathscr{A}$. Therefore, to prove $\mathscr{A}$ is a closed set it is sufficient only to prove that if $t^{*}>t$ and all $t$ fall in $\mathscr{A}$ then $t^{*}$ is also in $\mathscr{A}$. If the result is not true, there exist at least two distinct points $X_{1}, X_{2}$ in $B_{t^{*}}$ such that $f\left(X_{1}\right)=f\left(X_{2}\right)$. Because of $\left|X_{1}\right|<t^{*},\left|X_{2}\right|<t^{*}$, one can find a $t^{* *}$ satisfying $\left|X_{1}\right|<t^{* *}<t^{*}$, $\left|X_{2}\right|<t^{* *}<t^{*}$. The formulas above imply $X_{1}, X_{2} \in B_{t^{* *}}$. But $t^{* *} \in \mathscr{A}$. This yields $f\left(X_{1}\right) \neq f\left(X_{2}\right)$. It is impossible. So $\mathscr{A}$ is a closed set. Finally, we need to prove the set $\mathscr{A}$ is also an open set. For the end, only to verify that if $f$ is a one to one mapping on $B_{t}$ then there is a positive $\varepsilon$ small enough such that $f$ is also a one to one mapping on $B_{t+\varepsilon}$. If not, there is a sequence $\varepsilon_{n}>0$ with $\lim _{n \rightarrow \infty} \varepsilon_{n}=0$ such that one can find two sequences $\left\{X_{n}\right\},\left\{Y_{n}\right\}$ satisfying the following conditions:

$$
X_{n}, Y_{n} \in B_{t+\varepsilon_{n}}, \quad X_{n} \neq Y_{n}, \quad f\left(X_{n}\right)=f\left(Y_{n}\right)
$$


for all $n=1,2, \ldots$. Obviously,

$$
\lim _{n \rightarrow \infty}\left|Y_{n}\right|=\lim _{n \rightarrow \infty}\left|Y_{n}\right|=t \text {. }
$$

It is easy to see that $\left\{X_{n}\right\},\left\{Y_{n}\right\}$ are bounded sequences. So there exist two subsequences $\left\{X_{n_{k}}\right\},\left\{Y_{n_{k}}\right\}$ of $\left\{X_{n}\right\},\left\{Y_{n}\right\}$ which converge to $X$ and $Y$ respectively, that is

$$
\lim _{k \rightarrow \infty} X_{n_{k}}=X, \quad \lim _{k \rightarrow \infty} Y_{n_{k}}=Y .
$$

The two subsequences satisfy

$$
f\left(X_{n_{k}}\right)=f\left(Y_{n_{k}}\right), \quad X_{n_{k}} \neq Y_{n_{k}}, \quad \text { for } k=1,2, \ldots,
$$

and

$$
\lim _{k \rightarrow \infty}\left|X_{n_{k}}\right|=\lim _{k \rightarrow \infty}\left|Y_{n_{k}}\right|=t .
$$

Obviously, $X, Y \in \partial B_{t}$. If $X \neq Y$ that is contrary to the result in the second step. If $X=Y$ it implies that there are two points denoted still by $\left\{X_{n_{k}}\right\},\left\{Y_{n_{k}}\right\}$ in any neighborhood of $X=Y$ such that $X_{n_{k}} \neq Y_{n_{k}}$, but $f\left(X_{n_{k}}\right)=f\left(Y_{n_{k}}\right)$. It is obviously impossible due to the assumption of $f$ being local biholomorphic.

Combining the above argument we obtain $\mathscr{A}=[0,1]$. Hence $f$ is biholomorphic and $f$ is convex from the argument of the first step.

It is worth noting when $n=1$, the inequality (24) has the following form:

$$
|b|^{2}+\operatorname{Re}\left(b^{2}\left(\frac{d w}{d z}\right)^{2} \bar{z} \frac{d^{2} z}{d w^{2}}\right) \geq 0, \quad \operatorname{Re}(b \bar{z})=0 .
$$

Because of

$$
\frac{d^{2} w}{d z^{2}}\left(\frac{d z}{d w}\right)^{2}=-\frac{d w}{d z} \frac{d^{2} z}{d w^{2}}
$$

we have

$$
\begin{aligned}
& \text { (28) }|b|^{2}-\operatorname{Re}\left(\bar{z} b^{2} \frac{d w}{d z}\left(\frac{d z}{d w}\right)^{2} \frac{d^{2} w}{d z^{2}}\right)=|b|^{2}-\operatorname{Re}\left(\bar{z} b^{2} \frac{d^{2} w}{d z^{2}} \frac{d z}{d w}\right) \\
& =|b|^{2}+\operatorname{Re}\left(\bar{z} b^{2} \frac{f^{\prime \prime}}{f^{\prime}}\right) \geq 0 \text {. }
\end{aligned}
$$

Furthermore, from $\operatorname{Re}(b \bar{z})=b \bar{z}-\bar{b} z=0, \bar{z} b=-\bar{b} z$, the inequality (28) can be rewritten as

$$
|b|^{2}\left(1+\operatorname{Re} \frac{z f^{\prime \prime}}{f^{\prime}}\right) \geq 0 .
$$


It just is the necessary and sufficient condition of univalent convex functions defined on the unit disc in the complex plane $\mathbb{C}$.

In [6] K. Kikuchi stated the following theorem: Let $f$ be a holomorphic mapping from the ball $B$ into $\mathbb{C}^{n}$ with $f(0)=0$ and $\operatorname{det} J_{f}(Z) \neq 0, Z \in B$. Then $f$ is biholomorphic and convex if and only if

$$
\operatorname{Re}\left[|Z A|^{2}+\bar{Z} J_{f}^{-1}(Z) \widetilde{A}\right] \geq 0
$$

where

$$
\begin{aligned}
A= & \left(\begin{array}{cccc}
a_{1} & 0 & \cdots & 0 \\
0 & a_{2} & \cdots & 0 \\
\vdots & \vdots & \ddots & \vdots \\
0 & 0 & \cdots & a_{n}
\end{array}\right), \quad a_{j} \geq 0, \quad j=1,2, \ldots, n, \\
\tilde{A} & =\left(\begin{array}{l}
Z A \frac{\partial^{2} f_{1}}{\partial Z^{\prime} \partial Z}(Z) A^{\prime} Z^{\prime} \\
\cdots \cdots \cdots \cdots \\
Z A \frac{\partial^{2} \tilde{f}_{n}}{\partial Z^{\prime} \partial Z}(Z) A^{\prime} Z^{\prime}
\end{array}\right)
\end{aligned}
$$

with $Z A \neq 0$. The necessary and sufficient condition is equivalent to the one given by Suffridge in [7].

We quote a counter-example given by Taishun Liu in [4] to show that the condition (29) is not sufficient to conclude the section. Set

$$
f(Z)=\left(\frac{z_{1}}{1-z_{1}}, \frac{z_{2}}{1-z_{2}}, \ldots, \frac{z_{n}}{1-z_{n}}\right) .
$$

Clearly, the mapping is biholomorphic and a simple calculation gives

$$
J_{f}^{-1}(Z)=\left(\begin{array}{cccc}
\left(1-z_{1}\right)^{2} & 0 & \cdots & 0 \\
0 & \left(1-z_{2}\right)^{2} & \cdots & 0 \\
\vdots & \vdots & \ddots & \vdots \\
0 & 0 & \cdots & \left(1-z_{n}\right)^{2}
\end{array}\right)
$$

and $\partial^{2} f_{j} / \partial Z^{\prime} \partial Z$ is the matrix of which the unique non-zero element is $\frac{1}{\left(1-z_{j}\right)^{3}}$ at $j$ column and $j$ row.

Putting (30) into the left side of (29), we have

$$
\operatorname{Re}\left[|Z A|^{2}+\bar{Z} J_{f}^{-1}(Z) \widetilde{A}\right]=\operatorname{Re}\left[\sum_{j=1}^{n} a_{j}^{2}\left|z_{j}\right|^{2}\left(\frac{1+z_{j}}{1+z_{j}}\right)\right]>0 .
$$

Hence, according to the result by Kikuchi, the mapping

$$
f(Z)=\left(\frac{z_{1}}{1-z_{1}}, \frac{z_{2}}{1-z_{2}}, \ldots, \frac{z_{n}}{1-z_{n}}\right)
$$


should be convex. That is to say for $0<r<1$

$$
\frac{1}{n} \sum_{k=1}^{n} f\left(r e_{k}\right) \in\{f(B)\}
$$

where $e_{k}$ is the unit vector of which $k$ th component equals to 1 . So

$$
f^{-1}\left[\frac{1}{n} \sum_{k=1}^{n} f\left(r e_{k}\right)\right] \in B \text {. }
$$

But one has

$$
f^{-1}\left[\frac{1}{n} \sum_{k=1}^{n} f\left(r e_{k}\right)\right]=\left(\frac{r}{r+n(1-r)}, \ldots, \frac{r}{r+n(1-r)}\right) .
$$

When $r$ is close to 1 the right side of (31) does not belong to $B$. Therefore, the mapping $f$ is not convex.

4. Characteristics of the image of biholomorphic convex mappings. As an application of the theorems in $\S \S 1$ and 2, we now consider characteristics of the image of biholomorphic convex mappings from $B$ to $\mathbb{C}^{n}$.

THEOREM 3. Suppose that $f: B \rightarrow \mathbb{C}^{n}$ is a normalized biholomorphic convex mapping. Then

(1) $\frac{(1-\mu)^{5}}{\mu(1+\mu)^{3}} \leq$ principal curvature of $f\left(S_{\mu}\right) \leq \frac{(1+\mu)^{4}}{\mu(1-\mu)^{4}}$,

(2) $\quad \omega_{2 n-1} \frac{1-\mu}{2 \mu(1+\mu)} \int_{0}^{\mu} \frac{(1-t)^{n+1} t^{2 n-1}}{(1+t)^{3 n+3}} d t \leq$ volume of $f\left(S_{\mu}\right)$

$$
\leq n \omega_{2 n-1} \frac{1+\mu}{2 \mu(1-\mu)} \int_{0}^{\mu} \frac{(1+t)^{n+1} t^{2 n-1}}{(1-t)^{3 n+3}} d t,
$$

where $\omega_{2 n-1}$ is the volume of the unit sphere in $R^{2 n}$.

Proof. Fix $Z \in B-\{0\}$ and $b \in \mathbb{C}^{n}$ fulfilling $\operatorname{Re}\left(b^{\beta} \bar{z}^{\beta}\right)=0$. Let $\tilde{Z}=\frac{t Z}{|Z|}$ and $\tilde{b}=\frac{t b}{|Z|}, t \in D$. Then $\tilde{Z} \in B$ and $\operatorname{Re}\left(\tilde{b}^{\beta} \overline{\tilde{z}}^{\beta}\right)=0$. Since $W=f(Z)$ is a normalized biholomorphic convex mapping it follows from Theorem 2 that

$$
\frac{|t|^{2}|b|^{2}}{|Z|^{2}}+\operatorname{Re}\left\{\left.|t|^{2} \frac{t \bar{z}^{\alpha} b^{\beta} b^{\gamma}}{|Z|^{3}}\left(\frac{\partial w^{i}}{\partial z^{\beta}} \frac{\partial w^{j}}{\partial z^{\gamma}} \frac{\partial^{2} z^{\alpha}}{\partial w^{i} \partial w^{j}}\right)\right|_{\widetilde{Z}}\right\} \geq 0
$$

or

$$
\operatorname{Re}\left\{|b|^{2}+\left.\frac{t \bar{z}^{\alpha} b^{\beta} b^{\gamma}}{|Z|}\left(\frac{\partial w^{i}}{\partial z^{\beta}} \frac{\partial w^{j}}{\partial z^{\gamma}} \frac{\partial^{2} z^{\alpha}}{\partial w^{i} \partial w^{j}}\right)\right|_{\tilde{Z}}\right\} \geq 0
$$


The left side of (32) is a real-valued and nonnegative harmonic function. From Harnack's inequality we have

(33) $|b|^{2}\left(\frac{1-|t|}{1+|t|}\right) \leq|b|^{2}+\operatorname{Re}\left\{\left.\frac{t \bar{z}^{\alpha} b^{\beta} b^{\gamma}}{|Z|}\left(\frac{\partial w^{i}}{\partial z^{\beta}} \frac{\partial w^{j}}{\partial z^{\gamma}} \frac{\partial^{2} z^{\alpha}}{\partial w^{i} \partial w^{j}}\right)\right|_{\tilde{z}}\right\}$

$$
\leq|b|^{2}\left(\frac{1+|t|}{1-|t|}\right) \text {. }
$$

If we take $t=|Z|$, the formulae (33) becomes as follows:

$|b|^{2} \frac{1-|Z|}{1+|Z|} \leq|b|^{2}+\operatorname{Re}\left\{\bar{z}^{\alpha} b^{\beta} b^{\gamma}\left(\frac{\partial w^{i}}{\partial z^{\beta}} \frac{\partial w^{j}}{\partial z^{\gamma}} \frac{\partial^{2} z^{\alpha}}{\partial w^{i} \partial w^{j}}\right)\right\} \leq|b|^{2} \frac{1+|Z|}{1-|Z|}$

for any $Z \in B-\{0\}$ with $\operatorname{Re}\left(b^{\beta} \bar{z}^{\beta}\right)=0$. Now taking $b=a\left(\frac{\partial Z}{\partial W}\right)$ and noting (27) we have

$$
\begin{aligned}
a^{i} \frac{\partial z^{\beta}}{\partial w^{i}} \frac{\partial \bar{z}^{\beta}}{\partial \bar{w}^{j}} \bar{a}^{j}\left(\frac{1-|Z|}{1+|Z|}\right) & \leq|\nu| S(U, U) \\
& \leq a^{i} \frac{\partial z^{\beta}}{\partial w^{i}} \frac{\partial \bar{z}^{\beta}}{\partial \bar{w}^{j}} \bar{a}^{j}\left(\frac{1+|Z|}{1-|Z|}\right),
\end{aligned}
$$

where

$$
|\nu|=\sqrt{\bar{z}^{\alpha} \frac{\partial z^{\alpha}}{\partial w^{i}} \frac{\partial \bar{z}^{\beta}}{\partial \bar{w}^{i}} z^{\beta}}, \quad U=a^{i} \frac{\partial}{\partial w^{i}}+\bar{a}^{i} \frac{\partial}{\partial \bar{w}^{i}} .
$$

From Theorem 1, it follows that

$$
\begin{aligned}
& \delta_{i j} \geq\left(\frac{1-|Z|}{1+|Z|}\right)^{2}\left[\frac{1}{1-|Z|^{2}} \frac{\partial z^{\alpha}}{\partial \bar{w}^{i}} \frac{\partial \bar{z}^{\alpha}}{\partial \bar{w}^{j}}+\frac{1}{\left(1-|Z|^{2}\right)^{2}} \bar{z}^{\alpha} \frac{\partial z^{\alpha}}{\partial \bar{w}^{i}} \frac{\partial \bar{z}^{\beta}}{\partial \bar{w}^{j}} z^{\beta}\right], \\
& \delta_{i j} \leq\left(\frac{1+|Z|}{1-|Z|}\right)^{2}\left[\frac{1}{1-|Z|^{2}} \frac{\partial z^{\alpha}}{\partial \bar{w}^{i}} \frac{\partial \bar{z}^{\alpha}}{\partial \bar{w}^{j}}+\frac{1}{\left(1-|Z|^{2}\right)^{2}} \bar{z}^{\alpha} \frac{\partial z^{\alpha}}{\partial \bar{w}^{i}} \frac{\partial \bar{z}^{\beta}}{\partial \bar{w}^{j}} z^{\beta}\right] .
\end{aligned}
$$

Therefore, the inequalities

$$
\begin{aligned}
& |a|^{2} \geq \frac{1}{(1+|Z|)^{4}}\left[\left(1-|Z|^{2}\right) a^{i} \frac{\partial z^{\alpha}}{\partial w^{i}} \frac{\partial \bar{z}^{\alpha}}{\partial \bar{w}^{j}} \bar{a}^{j}+\left|a^{i} \bar{z}^{\alpha} \frac{\partial z^{\alpha}}{\partial w^{i}}\right|^{2}\right], \\
& |a|^{2} \leq \frac{1}{(1-|Z|)^{4}}\left[\left(1-|Z|^{2}\right) a^{i} \frac{\partial z^{\alpha}}{\partial w^{i}} \frac{\partial \bar{z}^{\alpha}}{\partial \bar{w}^{j}} \bar{a}^{j}+\left|a^{i} \bar{z}^{\alpha} \frac{\partial z^{\alpha}}{\partial w^{i}}\right|^{2}\right]
\end{aligned}
$$

hold for any $Z \in B$. By the Schwartz inequality

$$
0 \leq\left|a^{i} \bar{z}^{\alpha} \frac{\partial z^{\alpha}}{\partial w^{i}}\right|^{2} \leq|Z|^{2}\left|a^{i} \frac{\partial z^{\alpha}}{\partial w^{i}} \frac{\partial \bar{z}^{\alpha}}{\partial \bar{w}^{j}} \bar{a}^{j}\right|
$$


(34) can be read as

$$
\frac{1-|Z|}{(1+|Z|)^{3}} a^{i} \frac{\partial z^{\alpha}}{\partial w^{i}} \frac{\partial \bar{z}^{\alpha}}{\partial \bar{w}^{j}} \bar{a}^{j} \leq|a|^{2} \leq \frac{1}{(1-|Z|)^{4}} a^{i} \frac{\partial z^{\alpha}}{\partial w^{i}} \frac{\partial \bar{z}^{\alpha}}{\partial \bar{w}^{j}} \bar{a}^{j} .
$$

Substituting (36) into (34), we obtain

$$
\begin{aligned}
& |\nu| S(U, U) \geq \frac{(1-|Z|)^{5}}{1+|Z|}|U|^{2}, \\
& |\nu| S(U, U) \leq\left(\frac{1+|Z|}{1-|Z|}\right)^{4}|U|^{2} .
\end{aligned}
$$

On the other hand, again using Theorem 1 we get

$$
\left(\frac{1+|Z|}{1-|Z|}\right)^{2}\left(g_{\alpha \bar{\beta}}\right) \geq\left(\frac{\partial w^{i}}{\partial z^{\alpha}} \frac{\partial \bar{w}^{i}}{\partial \bar{z}^{\beta}}\right) \geq\left(\frac{1-|Z|}{1+|Z|}\right)^{2}\left(g_{\alpha \bar{\beta}}\right) \text {. }
$$

This yields

$$
\left(\frac{1-|Z|}{1+|Z|}\right)^{2}\left(g^{\alpha \bar{\beta}}\right) \leq\left(\frac{\partial z^{\alpha}}{\partial w^{i}} \frac{\partial \bar{z}^{\beta}}{\partial \bar{w}^{i}}\right) \leq\left(\frac{1+|Z|}{1-|Z|}\right)^{2}\left(g^{\bar{\beta} \alpha}\right),
$$

where $\left(g^{\bar{\beta}} \alpha\right)$ is the inverse matrix of $\left(g_{\alpha \bar{\beta}}\right)$. Hence

$$
\begin{gathered}
\frac{(1-|Z|)^{3}}{1+|Z|}\left(\delta_{\beta \alpha}-\bar{z}^{\beta} z^{\alpha}\right) \bar{z}^{\beta} z^{\alpha} \leq \bar{z}^{\alpha} \frac{\partial z^{\alpha}}{\partial w^{i}} \frac{\partial \bar{z}^{\beta}}{\partial \bar{w}^{j}} z^{\beta} \\
\leq \frac{(1+|Z|)^{3}}{1-|Z|}\left(\delta_{\beta \alpha}-\bar{z}^{\beta} z^{\alpha}\right) \bar{z}^{\beta} z^{\alpha} .
\end{gathered}
$$

So, we obtain

$$
|Z|^{2}(1-|Z|)^{4} \leq|\nu|^{2} \leq|Z|^{2}(1+|Z|)^{4} .
$$

Putting (38) into (37), we have

$$
\frac{(1-\mu)^{5}}{\mu(1+\mu)^{3}} \leq \text { principal curvature of } f\left(S_{\mu}\right) \leq \frac{(1+\mu)^{4}}{\mu(1-\mu)^{4}} \text {. }
$$

To yield the volume of the hypersurface $f\left(S_{\mu}\right)$ of $(2 n-1)$-dimension, we consider the function $r^{2}=|Z|^{2} \circ f^{-1}$. Denote $\Delta=4 \partial^{2} / \partial w^{i} \partial \bar{w}^{i}$. Then we have

$$
\begin{aligned}
\int_{f\left(\beta_{\mu}\right)} \frac{\Delta r^{2}}{1-r^{2}} d W= & -\int_{f\left(B_{\mu}\right)} \frac{\left|\nabla r^{2}\right|}{\left(1-r^{2}\right)^{2}} d W \\
& +\frac{1}{1-\mu^{2}} \int_{\partial f\left(B_{\mu}\right)} \frac{\partial r^{2}}{\partial n} d \sigma,
\end{aligned}
$$


where $d W$ is the volume element of $\mathbb{C}^{n}=\mathscr{R}^{2 n}, d \sigma$ that of $\partial f\left(B_{\mu}\right)$ and $n=\frac{\nu}{|\nu|}$ the unit normal vector. Because of

$$
\frac{\partial r^{2}}{\partial n}=\frac{\nu}{|\nu|} r^{2}=\frac{2}{|\nu|}\left(\bar{z}^{\alpha} \frac{\partial z^{\alpha}}{\partial w^{i}} \frac{\partial \bar{z}^{\beta}}{\partial \bar{w}^{i}} z^{\beta}\right)=2|\nu|
$$

(39) becomes

(40)

$$
\int_{f\left(B_{\mu}\right)} \frac{\left(1-r^{2}\right) \Delta r^{2}+\left|\nabla r^{2}\right|^{2}}{\left(1-r^{2}\right)^{2}} d W=\frac{2}{1-\mu^{2}} \int_{f\left(S_{\mu}\right)}|\nu| d \sigma .
$$

On the other hand, we have

$$
\Delta r^{2}=4 \frac{\partial z^{\alpha}}{\partial w^{i}} \frac{\partial \bar{z}^{\beta}}{\partial \bar{w}^{i}}, \quad\left|\nabla r^{2}\right|^{2}=4 \bar{z}^{\alpha} \frac{\partial z^{\alpha}}{\partial w^{i}} \frac{\partial \bar{z}^{\beta}}{\partial \bar{w}^{i}} z^{\beta} .
$$

Hence

$$
\frac{\left(1-r^{2}\right) \Delta r^{2}+\left|\nabla r^{2}\right|^{2}}{\left(1-r^{2}\right)^{2}}=g_{\alpha \bar{\beta}} \frac{\partial z^{\alpha}}{\partial w^{i}} \frac{\partial \bar{z}^{\beta}}{\partial \bar{w}^{i}}
$$

Putting (41) into (40) we obtain

$$
\int_{f\left(B_{\mu}\right)} g_{\alpha \bar{\beta}} \frac{\partial z^{\alpha}}{\partial w^{i}} \frac{\partial \bar{z}^{\beta}}{\partial \bar{w}^{i}} d W=\frac{2}{1-\mu^{2}} \int_{f\left(S_{\mu}\right)}|\nu| d \sigma .
$$

From Theorem 1 we have

$$
\left(\frac{1+|Z|}{1-|Z|}\right)^{2} g_{\alpha \bar{\beta}} \frac{\partial z^{\alpha}}{\partial w^{i}} \frac{\partial \bar{Z}^{\beta}}{\partial \bar{w}^{i}} \geq n \geq\left(\frac{1-|Z|}{1+|Z|}\right)^{2} g_{\alpha \bar{\beta}} \frac{\partial z^{\alpha}}{\partial w^{i}} \frac{\partial \bar{z}^{\beta}}{\partial \bar{w}^{i}}
$$

Substituting (43) into (42), we obtain

$$
\begin{aligned}
n \int_{f\left(B_{\mu}\right)}\left(\frac{1-r}{1+r}\right)^{2} d W & \leq \frac{2}{1-\mu^{2}} \int_{f\left(S_{\mu}\right)}|\nu| d \sigma \\
& \leq n \int_{f\left(B_{\mu}\right)}\left(\frac{1+r}{1-r}\right)^{2} d W
\end{aligned}
$$

Combining (39) with (44), we obtain

$$
\begin{aligned}
& \frac{n(1-\mu)}{2 \mu(1+\mu)} \int_{f\left(B_{\mu}\right)}\left(\frac{1-r}{1+r}\right)^{2} d W \\
& \quad \leq \int_{f\left(S_{\mu}\right)} d \sigma \leq \frac{n(1+\mu)}{2 \mu(1-\mu)} \int_{f\left(B_{\mu}\right)}\left(\frac{1+r}{1-r}\right)^{2} d W
\end{aligned}
$$


or

$$
\begin{aligned}
& \int_{f\left(S_{\mu}\right)} d \sigma \geq \frac{n(1-\mu)}{2 \mu(1+\mu)} \int_{B_{\mu}}\left(\frac{1-|Z|}{1+|Z|}\right)^{2}\left|\operatorname{det} J_{f}(Z)\right|^{2} d Z, \\
& \int_{f\left(S_{\mu}\right)} d \sigma \leq \frac{n(1+\mu)}{2 \mu(1-\mu)} \int_{B_{\mu}}\left(\frac{1+|Z|}{1-|Z|}\right)^{2}\left|\operatorname{det} J_{f}(Z)\right|^{2} d Z,
\end{aligned}
$$

where $d Z$ is the volume element of $\mathbb{C}^{n}=\mathscr{R}^{2 n}$. Finally, again using Theorem 1 we obtain

$$
\frac{(1+|Z|)^{n-1}}{(1-|Z|)^{3 n+1}} \geq\left|\operatorname{det} J_{f}(Z)\right|^{2} \geq \frac{(1-|Z|)^{n-1}}{(1+|Z|)^{3 n+1}}
$$

Putting (46) into (45) concludes Theorem 3.

\section{REFERENCES}

[1] H. Cartan, Sur la Possibilité d'étendre aux Fonctions de Plusieurs Variables Complexes la Theorié des Fonctions Univalentes, Note to P. Montel, Leçons sur les fonctions univalentes ou multivalentes, Gauthier-Villars, Paris, 1933, 129155.

[2] P. Duren and W. Rudin, Distortion in several variables, Complex Analysis, Theory and Application, 5 (1986), 323-326.

[3] C. H. FitzGerald, S. Gong and R. W. Bernard, Distortion theorem for biholomorphic mappings in $\mathbb{C}^{2}$, Chinese Sci. Bull., 5 (1990), 353-356.

[4] Taishun Liu, The Growth Theorems, Covering Theorems and Distortion Theorems in Several Complex Variables, Doctoral thesis, 1989 (in Chinese).

[5] S. Gong and X. A. Zheng, Distortion theorems for biholomorphic mappings in transitive domains (II), Chinese Sci. Bull., 5 (1990), 330-332 (in Chinese).

[6] K. Kikuchi, Starlike and convex mappings in several complex variables, Pacific J. Math., 2 (1973), 569-880.

[7] T. J. Suffridge, The principle of subordination applied to functions of several variables, Pacific J. Math., 33 (1970), 241-248.

[8] S. I. Pinchuk, Holomorphic Maps in $\mathbb{C}^{n}$ and the Problem of Holomorphic Equivalence, Several Complex Variables III, G. M. Khenkin (Ed.), Springer-Verlag, 1989.

[9] S. Gong, S. K. Wang and Q. Yu, The growth and 1/4-theorems for starlike mappings in $B^{p}$, Chinese Ann. Math., 11 B 1 (1990), 100-104.

Received October 29, 1991 and in revised form April 29, 1992. Supported by National Natural Science Foundation of China.

ACADEMIa Sinica

P.O. BoX 2734

BeIJing 100080 China 


\title{
PACIFIC JOURNAL OF MATHEMATICS
}

Founded by

\author{
E. F. BECKenBaCH (1906-1982) F. WolF (1904-1989)
}

\section{EDITORS}

Sun-Yung A. Chang

(Managing Editor)

University of California

Los Angeles, CA 90024-1555

chang@math.ucla.edu

F. Michael Christ

University of California

Los Angeles, CA 90024-1555

christ@math.ucla.edu

Herbert Clemens

University of Utah

Salt Lake City, UT 84112

clemens@math.utah.edu

\author{
R. FInN
}

THOMAS ENRIGHT

University of California, San Diego

La Jolla, CA 92093

tenright@ucsd.edu

Nicholas ERColani

University of Arizona

Tucson, AZ 85721

ercolani@math.arizona.edu

Stanford University

Stanford, CA 94305

finn@gauss.stanford.edu

VAUghan F. R. JoNES

University of California

Berkeley, CA 94720

vfr@math.berkeley.edu

\section{SUPPORTING INSTITUTIONS}

STEVEN KeRCKHOFF

Stanford University

Stanford, CA 94305

spk@gauss.stanford.edu

MARTIN ScharLemanN

University of California

Santa Barbara, CA 93106

mgscharl@henri.ucsb.edu

HAROLd STARK

University of California, San Diego La Jolla, CA 92093

V. S. VARADARAJAN

University of California

Los Angeles, CA 90024-1555

vsv@math.ucla.edu

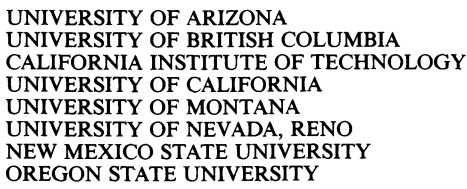

The Supporting Institutions listed above contribute to the cost of publication of this Journal, but they are not owners or publishers and have no responsibility for its content or policies.

Mathematical papers intended for publication in the Pacific Journal of Mathematics should be in typed form or offset-reproduced (not dittoed), double spaced with large margins. Please do not use built up fractions in the text of the manuscript. However, you may use them in the displayed equations. Underline Greek letters in red, German in green, and script in blue. The first paragraph must be capable of being used separately as a synopsis of the entire paper. In particular it should contain no bibliographic references. Please propose a heading for the odd numbered pages of less than 35 characters. Manuscripts, in triplicate, may be sent to any one of the editors. Please classify according to the 1991 Mathematics Subject Classification scheme which can be found in the December index volumes of Mathematical Reviews. Supply name and address of author to whom proofs should be sent. All other communications should be addressed to the managing editor, or Julie Honig, University of California, Los Angeles, California 90024-1555.

There are page-charges associated with articles appearing in the Pacific Journal of Mathematics. These charges are expected to be paid by the author's University, Government Agency or Company. If the author or authors do not have access to such Institutional support these charges are waived. Single authors will receive 75 free reprints; joint authors will receive a total of 100 free reprints. Additional copies may be obtained at cost in multiples of 50 .

The Pacific Journal of Mathematics (ISSN 0030-8730) is published monthly except for July and August. Regular subscription rate: $\$ 200.00$ a year (10 issues). Special rate: $\$ 100.00$ a year to individual members of supporting institutions.

Subscriptions, orders for numbers issued in the last three calendar years, and changes of address should be sent to Pacific Journal of Mathematics, P.O. Box 969, Carmel Valley, CA 93924, U.S.A. Old back numbers obtainable from Kraus Periodicals Co., Route 100, Millwood, NY 10546.

The Pacific Journal of Mathematics at P.O. Box 969, Carmel Valley, CA 93924 (ISSN 0030-8730) is published monthly except for July and August. Second-class postage paid at Carmel Valley, California 93924, and additional mailing offices. Postmaster: send address changes to Pacific Journal of Mathematics, P.O. Box 969, Carmel Valley, CA 93924.

PUBLISHED BY PACIFIC JOURNAL OF MATHEMATICS, A NON-PROFIT CORPORATION

This publication was typeset using $\mathcal{A} \mathcal{M S}-\mathrm{T}_{\mathrm{E}} \mathrm{X}$, the American Mathematical Society's $\mathrm{T}_{\mathrm{E}} \mathrm{X}$ macro system. Copyright (c) 1993 by Pacific Journal of Mathematics 


\section{PACIFIC JOURNAL OF MATHEMATICS}

Volume $161 \quad$ No. $2 \quad$ December 1993

On the method of constructing irreducible finite index subfactors of 201 Popa

FLORIN PETRE BOCA

Brownian motion and the heat semigroup on the path space of a

233 compact Lie group

JAY BARRY EPPERSON and TERRY M. LOHRENZ

Horizontal path spaces and Carnot-Carathéodory metrics

255

ZHONG GE

Biholomorphic convex mappings of ball in $\mathbb{C}^{n}$

SHENG GONG, SHI KUN WANG and QI HUANG YU

The Temperley-Lieb algebra at roots of unity

FREDERICK MiCHAEL GOODMAN and HANS WENZL

Jordan analogs of the Burnside and Jacobson density theorems

LUZIUS GRÜNENFELDER, M. OLMLADIČ and HEYDAR RADJAVI

Elliptic representations for $\mathrm{Sp}(2 n)$ and $\mathrm{SO}(n)$

REBECCA A. Herb

Reflexivity of subnormal operators

JOHN MCCARTHY

Knotting trivial knots and resulting knot types

KiMiHiko MOTEGI

Commutativity of selfadjoint operators

MitsURU UCHIYAMA

Correction to: "One-dimensional Nash groups" 